\title{
Graphs Associated with the Ideals of a Numerical Semigroup Having Metric Dimension 2
}

\author{
Ying Wang, ${ }^{1,2}$ Muhammad Ahsan Binyamin $\left(\mathbb{D},{ }^{3}\right.$ Wajid Ali, ${ }^{3}$ Adnan Aslam $\mathbb{D},{ }^{4}$ \\ and Yongsheng Rao ${ }^{2}{ }^{2}$ \\ ${ }^{1}$ Department of Network Technology, South China Institute of Software Engineering, Guangzhou, China \\ ${ }^{2}$ Institute of Computing Science and Technology, Guangzhou University, Guangzhou 510006, China \\ ${ }^{3}$ Department of Mathematics, GC University, Faisalabad, Pakistan \\ ${ }^{4}$ Department of Natural Sciences and Humanities, University of Engineering and Technology, Lahore (RCET), Pakistan
}

Correspondence should be addressed to Muhammad Ahsan Binyamin; ahsanbanyamin@gmail.com

Received 18 December 2020; Revised 7 January 2021; Accepted 23 January 2021; Published 9 February 2021

Academic Editor: Ali Ahmad

Copyright ( $\odot 2021$ Ying Wang et al. This is an open access article distributed under the Creative Commons Attribution License, which permits unrestricted use, distribution, and reproduction in any medium, provided the original work is properly cited.

Let $\Lambda$ be a numerical semigroup and $I \subset \Lambda$ be an irreducible ideal of $\Lambda$. The graph $G_{I}(\Lambda)$ assigned to an ideal $I$ of $\Lambda$ is a graph with elements of $(\Lambda \backslash I)^{*}$ as vertices, and any two vertices $x$ and $y$ are adjacent if and only if $x+y \in I$. In this work, we give a complete characterization (up to isomorphism) of the graph $G_{I}(\Lambda)$ having metric dimension 2.

\section{Introduction}

In algebraic combinatorics, the study of graphs associated with algebraic objects is one of the most important and fascinating fields of research. During the last couple of decades, a lot of research is carried out in this field. There are many papers on assigning graphs to rings, groups, and semigroups [1-6]. Several authors [7-13] studied different properties of these graphs including diameter, girth, domination, metric dimension, central sets, and planarity.

We start by defining some basic concept related to graph theory. A graph $G=(V(G), E(G))$ has a vertex set $V(G)$ and the edge set $E(G)$. The cardinality of the vertex set and edge set is called the order and size of $G$, respectively. A path in $G$ is a sequence of edges $u_{1} u_{2}, u_{2} u_{3}, \ldots, u_{k-1} u_{k}$. A graph $G$ is connected if every pair of vertices $x, y \in V(G)$ is connected by a path. The distance between two vertices $x, y \in V(G)$ is denoted by $d(x, y)$ and is the length of the shortest path between them. The diameter of $G$ is denoted by $d(G)$ and is defined as the largest distance between the vertices of $G$. Let $U=\left\{u_{1}, u_{2}, \ldots, u_{r}\right\}$ be an ordered subset of $V(G)$. Then, the $r$-tuple $\left(d\left(u, u_{1}\right), d\left(u, u_{2}\right), \ldots, d\left(u, u_{r}\right)\right)$ is the representation $u$ with respect to $U$. The vertex $u$ is said to be resolved by $U$ if $\left(d\left(u, u_{1}\right), d\left(u, u_{2}\right), \ldots, d\left(u, u_{r}\right) \neq\left(d\left(v, u_{1}\right), d\left(v, u_{2}\right), \ldots\right.\right.$, $\left.\left.d\left(v, u_{r}\right)\right)\right)$ for any vertex $v \in V(G)$. The set $U$ is called resolving set of $G$ if distinct vertices of $G$ have distinct representations with respect to $U$, and it is called basis of $G$ if it is a resolving set with minimal cardinality. The metric dimension of $G$, denoted by $\mu(G)$, is the cardinality of basis. The concept of metric dimension was introduced by Slater [14] and later studied by Harary and Melter [15]. It has many applications, for example, robot navigation [16], pharmaceutical chemistry $[17,18]$, sonar and coast guard long range navigation [14], and combinatorial optimization [19].

Let $\mathbb{N}$ be set of nonnegative integers. A subset $\Lambda \subset \mathbb{N}$ is said to be numerical semigroup if the following holds:

(1) $0 \in \Lambda$

(2) $x+y \in \Lambda$ for all $x, y \in \Lambda$

(3) $\mathbb{N} \backslash \Lambda$ is finite

It is easy to observe that the numerical semigroup is a commutative monoid. Thus, the set of numerical semigroups classifies the set of all submonoids of $(\mathbb{N},+)$. The elements of the set $\mathbb{N} \backslash \Lambda$ are called gaps of $\Lambda$, and the largest element of this set is known as Frobenius number. Note that every numerical semigroup is finitely generated; that is, there exist a set $A=\left\{a_{1}, a_{2}, \ldots, a_{t}\right\}$ such that $\Lambda=\langle A\rangle=\left\{n_{1} a_{1}+\ldots\right.$ 
$\left.n_{t} a_{t}: n_{1}, \ldots n_{t} \in \mathbb{N}\right\}$. Moreover, every numerical semigroup has a unique minimal system of generators. The cardinality of the minimal system of generators is called embedding dimension of $\Lambda$. It is denoted by $e_{\Lambda}$. A subset $I$ of numerical semigroup $\Lambda$ is ideal (integral ideal) of $\Lambda$ if for all $x \in I$ and $s \in \Lambda$ and the element $x+s \in I$. An ideal $I$ is called irreducibly ideal if it cannot be written as intersections of two or more than two ideals which contained it properly. For more details on theory of numerical semigroup, the interested readers can refer to [20].

Recently, several authors studied the metric dimension of the graphs associated with the algebraic objects. Soleymanivarniab et al. [21] gave some metric dimension formula for annihilator graphs. Bailey et al. [22] studied the constructions of resolving sets of Kneser and Johnson graphs and provided bounds on their metric dimension. Faisal et al. [23] studied the metric dimension of the commuting graph of a dihedral group. The metric dimension of a zero-divisor graph of a commutative ring was studied in [13], while the metric dimension of a total graph of a finite commutative ring was studied in [24]. For more results on the metric dimension, we refer the readers to [25-30].

\section{Notation and Preliminaries}

Let $\Lambda=\langle\mathscr{A}\rangle$ be a numerical semigroup, where $\mathscr{A}=\left\{a_{1}, a_{2}, \ldots, a_{n}\right\}$ is the minimal system of generators of $\Lambda$. Then, every $x \in \Lambda$ has a representation of the form $u_{1} a_{1}+u_{2} a_{2}+\cdots+u_{n} a_{n}$, where $u_{1}, u_{2}, \ldots, u_{n}$ are nonnegative integers. Let $1 \leq p \leq n$ be a fixed integer. We say that an element $x \in \Lambda$ has a $p$-representation if there exist $a_{i_{1}}, a_{i_{2}}, \ldots, a_{i_{p}} \in \mathscr{A}$ and $u_{i_{1}}, u_{i_{2}}, \ldots, u_{i_{p}}$ positive integers such that $x=u_{i_{1}} a_{i_{1}}+u_{i_{2}} a_{i_{2}}+\cdots+u_{i_{p}} a_{i_{p}}$; that is, $x$ can be written as linear combination of exactly $p$ generator of $\Lambda$. Let $\Lambda_{p}$ denote the set containing all the elements $x \in \Lambda$, which have a $p$ representation. It is easy to see that

$$
\Lambda=\bigcup_{p=1}^{n} \Lambda_{p}
$$

Note that an element $x \in \Lambda$ may have more than one $p$ representations. For an element $x \in \Lambda_{p}$, we use the notation $\Sigma_{p}$ if it has a unique $p$ representation and $\Sigma_{p, 1}, \Sigma_{p, 2}, \ldots, \Sigma_{p, r}$ if it has $r$ number of $p$ representations. Let $\Sigma_{p} \in \Lambda_{p}$, then there exist two $p$-tuples, the coefficients $p$ tuple $\left(u_{i_{1}}, u_{i_{2}}, \ldots, u_{i_{p}}\right) \in \mathbb{Z}_{>0}^{p}$, and the generators $p$-tuple $\left(a_{i_{1}}, a_{i_{2}}, \ldots, a_{i_{p}}\right) \in \mathbb{Z}_{>0}^{p}$ such that $\Sigma_{p}=u_{i_{1}} a_{i_{1}}+u_{i_{2}} a_{i_{2}}+\cdots+$ $u_{i_{p}} a_{i_{p}}$. We denote the coefficient and generators $p$ tuple of an element $\Sigma_{p}$ by $c\left(\Sigma_{p}\right)$ and $g\left(\Sigma_{p}\right)$, respectively. Also, the $j$-th component of $c\left(\Sigma_{p}\right)$ and $g\left(\Sigma_{p}\right)$ is denoted by $c_{j}\left(\Sigma_{p}\right)$ and $g_{j}\left(\Sigma_{p}\right)$, respectively. By using the above notations, for any $x \in \Lambda$, we define

$$
\begin{aligned}
\Lambda_{p}(x) & =\left\{\Sigma_{p}: \Sigma_{p}=x\right\}, \\
\Lambda(x) & =\bigcup_{p=1}^{n} \Lambda_{p}(x) .
\end{aligned}
$$
we set
For a $p$-representation $\Sigma_{p}=u_{i_{1}} a_{i_{1}}+u_{i_{2}} a_{i_{2}}+\cdots+u_{i_{p}} a_{i_{p}}$,

$$
\mathscr{B}\left(\Sigma_{p}\right)=\left\{v_{i_{1}} a_{i_{1}}+v_{i_{2}} a_{i_{2}}+\cdots+v_{i_{p}} a_{i_{p}}: 0 \leq v_{i_{j}} \leq u_{i_{j}}, \quad 1 \leq j \leq p\right\} .
$$

Lemma 1. With the notations defined above, we have

$$
\mathscr{B}(x)=\bigcup_{\Sigma_{p} \in \Lambda(x)} \mathscr{B}\left(\Sigma_{p}\right) .
$$

Proof. The proof of this lemma follows from the definition of $\mathscr{B}(x)$.

Let $\Lambda$ be a numerical semigroup and $I \subset \Lambda$ be irreducible ideal of $\Lambda$. Binyamin et al. [31] assigned a graph to numerical semigroup $\Lambda$ and studied its properties. Peng Xu et al. [32] assign a graph $G_{I}(\Lambda)$ to the ideal $I$ of numerical semigroup $\Lambda$ with vertex set $V\left(G_{I}(\Lambda)\right)=(\Lambda \backslash I)^{*}$ and two vertices $x, y$ are adjacent if and only if $x+y \in I$. Barucci [33] showed that every irreducible ideal $I$ of numerical semigroup $\Lambda$ can be expressed in the form $\Lambda \backslash B(x)$, where $B(x)=\{y \in \Lambda$ : $x-y \in \Lambda\}$, for some $x \in \Lambda$. Hence, the vertex set of the graph $G_{I}(\Lambda)$ is the set $\left\{v_{i}: i \in B^{*}(x)\right\}$ for some $x \in \Lambda$. Peng $\mathrm{Xu}$ et al. [32] proved that the graph $G_{I}(\Lambda)$ is always connected and diameter 2 . The aim of this paper is to find all the graphs $G_{I}(\Lambda)$ having metric dimension 2 . The following result by Chartrand et al. [18] gives bound on the order of graph with given metric dimension $k$ and diameter $d$.

Theorem 1. Let $G$ be a graph with metric dimension $k$ and $|V(G)|=n$. Let $d$ be the diameter of $G$. Then, $|V(G)| \leq d^{k}+k$.

Hence, to find graphs $G_{I}(\Lambda)$ with metric dimension 2 , it is enough to classify all graphs $G_{I}(\Lambda)$ of order less than or equal to 6 . In the next section, we give bounds for the graphs $G_{I}(\Lambda)$ of orders 4 and 5 .

\subsection{Bounds for the Graphs $G_{I}(\Lambda)$ of Orders 4 and 5}

Lemma 2. Let $\Lambda=\langle\mathscr{A}\rangle$ be a numerical semigroup of embedding dimension $n \geq 2$. Then, $\left|G_{I}(\Lambda)\right| \neq 4$, if one of the following holds:

(1) $\Lambda_{p}(x) \neq \varnothing$ for some $p \geq 3$.

(2) $\left|\Lambda_{1}(x)\right| \geq 3$.

(3) $\left|\Lambda_{2}(x)\right| \geq 2$.

(4) $\Lambda_{1}(x)=\varnothing$ and $\left|\Lambda_{2}(x)\right|=1$.

(5) $\left|\Lambda_{1}(x)\right|=2$ and $\left|\Lambda_{2}(x)\right|=1$.

Proof

(1) If $\Lambda_{p}(x) \neq \varnothing$ for some $p \geq 3$, then there is a $p$-representation $\Sigma_{p}$ of $x$ in $\Lambda_{p}(x)$. This gives $g_{1}\left(\Sigma_{p}\right), g_{2}\left(\Sigma_{p}\right), g_{3}\left(\Sigma_{p}\right), g_{1}\left(\Sigma_{p}\right)+g_{2}\left(\Sigma_{p}\right), g_{1}\left(\Sigma_{p}\right)+$ $g_{3}\left(\Sigma_{p}\right), g_{2} \quad\left(\Sigma_{p}\right)+g_{3}\left(\Sigma_{p}\right), x \in \mathscr{B}^{*} \quad\left(\Sigma_{p}\right) \subseteq \mathscr{B}^{*}(x)$. This implies $\left|G_{I}(\Lambda)\right| \neq 4$. 
(2) If $\left|\Lambda_{1}(x)\right| \geq 3$, then there are $\Sigma_{1,1}, \Sigma_{1,2}, \ldots$, $\Sigma_{1, r} \in \Lambda_{1}(x)$ with $r \geq 3$. We assume that $g\left(\Sigma_{1,1}\right)<$ $g\left(\Sigma_{1,2}\right)<\cdots<g\left(\Sigma_{1, r}\right)$ and then $c\left(\Sigma_{1,1}\right) \geq 5$. This gives $g\left(\Sigma_{1,1}\right), 2 g\left(\Sigma_{1,1}\right), 3 g\left(\Sigma_{1,1}\right), 4 g\left(\Sigma_{1,1}\right), 5 g\left(\Sigma_{1,1}\right)$ $\in \mathscr{B}^{*}\left(\Sigma_{1,1}\right)$, and therefore, $\left|G_{I}(\Lambda)\right| \neq 4$.

(3) If $\left|\Lambda_{2}(x)\right| \geq 2$, then we have $\Sigma_{2,1}, \Sigma_{2,2}, \ldots$, $\Sigma_{2, s} \in \Lambda_{2}(x)$ with $s \geq 2$. One can easily see that $\mathscr{B}^{*}\left(\Sigma_{2,1}\right) \cup \mathscr{B}^{*}\left(\Sigma_{2,2}\right) \cup \ldots \cup \mathscr{B}^{*}\left(\Sigma_{2, s}\right)$ must contain $g_{1}\left(\Sigma_{2,1}\right), \quad g_{2}\left(\Sigma_{2,1}\right), \quad g_{1}\left(\Sigma_{2,2}\right), \quad g_{2}\left(\Sigma_{2,2}\right), \quad g_{1}\left(\Sigma_{2,1}\right)+$ $g_{2}\left(\Sigma_{2,1}\right)$ and $g_{1}\left(\Sigma_{2,2}\right)+g_{2}\left(\Sigma_{2,2}\right)$. Therefore, $\left|G_{I}(\Lambda)\right| \neq 4$.

(4) Lemma 1: If $\Lambda_{1}(x)=\varnothing$ and $\left|\Lambda_{2}(x)\right|=1$ then there is the unique 2-representation $\Sigma_{2}$ of $x$. Now if $\Lambda_{p}(x) \neq \varnothing$ for some $p \geq 3$ then from (1), it follows that $\left|G_{I}(\Lambda)\right| \neq 4$, and if $\Lambda_{p}(x)=\varnothing$ for all $p \geq 3$, then gives $\mathscr{B}^{*}(x)=\mathscr{B}^{*}\left(\Sigma_{2}\right)$. So if $c\left(\Sigma_{2}\right)=(1,1)$, then $\left|G_{I}(\Lambda)\right|=3$; otherwise, $\left|G_{I}(\Lambda)\right|>4$. Consequently, $\left|G_{I}(\Lambda)\right| \neq 4$.

(5) If $\left|\Lambda_{1}(x)\right|=2$ and $\left|\Lambda_{2}(x)\right|=1$, then we can assume $\Sigma_{1,1}, \Sigma_{1,2} \in \Lambda_{1}(x)$ and $\Sigma_{2} \in \Lambda_{2}(x)$. This gives $g\left(\Sigma_{1,1}\right), g\left(\Sigma_{1,2}\right), g_{1}\left(\Sigma_{2}\right), g_{2}\left(\Sigma_{2}\right), g_{1}\left(\Sigma_{2}\right)+g_{2}\left(\Sigma_{2}\right)$ are in $\mathscr{B}^{*}(x)$, and therefore, $\left|G_{I}(\Lambda)\right| \neq 4$.

Lemma 3. Let $\Lambda=\langle\mathscr{A}\rangle$ be a numerical semigroup of embedding dimension $n \geq 2$. Then, $\left|G_{I}(\Lambda)\right| \neq 5$, if one of the following holds:

(1) $\Lambda_{p}(x) \neq \varnothing$ for some $p \geq 3$.

(2) $\left|\Lambda_{1}(x)\right| \geq 2$.

(3) $\left|\Lambda_{2}(x)\right| \geq 3$.

(4) $\left|\Lambda_{1}(x)\right|=1$ and $\left|\Lambda_{2}(x)\right|=2$.
Proof. This lemma can be proved in a similar way as we proved Lemma 2.

2.2. Computation of Irreducible Ideals for the Graphs $G_{I}(\Lambda)$ of Orders 4 and 5

Lemma 4. Let $\Lambda=\langle\mathscr{A}\rangle$ be a numerical semigroup of embedding dimension $n \geq 2$. If $\left|G_{I}(\Lambda)\right|=4$, then $x$ is one of the following:

(1) $x=4 g\left(\Sigma_{1}\right)$.

(2) $x=3 g\left(\Sigma_{1,1}\right)$ and $x=2 g\left(\Sigma_{1,2}\right)$.

(3) $x=2 g\left(\Sigma_{1}\right)$ and $x=g_{1}\left(\Sigma_{2}\right)+g_{2}\left(\Sigma_{2}\right)$.

Proof. If $\left|G_{I}(\Lambda)\right|=4$, then from Lemma 2, it follows that $x \in \Lambda$ satisfies one of the following conditions:

$$
\begin{aligned}
& \left|\Lambda_{1}(x)\right| \leq 2 \text { and } \Lambda_{p}(x)=\varnothing, \forall p \geq 2 . \\
& \left|\Lambda_{1}(x)\right|=1,\left|\Lambda_{2}(x)\right|=1 \text { and } \Lambda_{p}(x)=\varnothing, \forall p \geq 3 .
\end{aligned}
$$

If $\left|\Lambda_{1}(x)\right|=1$ and $\Lambda_{p}(x)=\varnothing, \forall p \geq 2$, then $x$ has a unique 1-representation $\Sigma_{1}$. By Lemma 1, we get $\mathscr{B}^{*}(x)=\mathscr{B}^{*}\left(\Sigma_{1}\right)=\left\{g\left(\Sigma_{1}\right), 2 g\left(\Sigma_{1}\right), \ldots, c\left(\Sigma_{1}\right) \cdot g\left(\Sigma_{1}\right)\right\}$. As $\left|G_{I}(\Lambda)\right|=4$, it follows that $c\left(\Sigma_{1}\right)=4$. This gives case (1).

Now if $\left|\Lambda_{1}(x)\right|=2$ and $\Lambda_{p}(x)=\varnothing, \forall p \geq 2$, then there are exactly two 1-representations, say $\Sigma_{1,1}$ and $\Sigma_{1,2}$ of $x$. Assume that $g\left(\Sigma_{1,1}\right)<g\left(\Sigma_{1,2}\right)$, then $c\left(\Sigma_{1,2}\right)<c\left(\Sigma_{1,1}\right)$ and $c\left(\Sigma_{1,1}\right)$ is not a multiple of $c\left(\Sigma_{1,2}\right)$. Then, it follows from Lemma 1 that

$$
\begin{aligned}
\mathscr{B}^{*}(x) & =\mathscr{B}^{*}\left(\Sigma_{1,1}\right) \cup \mathscr{B}^{*}\left(\Sigma_{1,2}\right)=\left\{g\left(\Sigma_{1,1}\right), 2 g\left(\Sigma_{1,1}\right), \ldots, c\left(\Sigma_{1,1}\right) \cdot g\left(\Sigma_{1,1}\right)\right\} \cup\left\{g\left(\Sigma_{1,2}\right), 2 g\left(\Sigma_{1,2}\right) \ldots, c\left(\Sigma_{1,2}\right) \cdot g\left(\Sigma_{1,2}\right)\right\} \\
& =\left\{g\left(\Sigma_{1,1}\right), 2 g\left(\Sigma_{1,1}\right), \ldots, c\left(\Sigma_{1,1}\right) \cdot g\left(\Sigma_{1,1}\right), g\left(\Sigma_{1,2}\right), 2 g\left(\Sigma_{1,2}\right), \ldots, c\left(\Sigma_{1,2}\right) \cdot g\left(\Sigma_{1,2}\right)\right\} .
\end{aligned}
$$

We show that $\left\{\mathscr{B}^{*}\left(\Sigma_{1,1}\right) \cap \mathscr{B}^{*}\left(\Sigma_{1,2}\right)\right\} \backslash\left\{c\left(\Sigma_{1,2}\right) \cdot g\right.$ $\left.\left(\Sigma_{1,2}\right)\right\}=\varnothing$. Let $p \cdot g\left(\Sigma_{1,1}\right)=q . g\left(\Sigma_{1,2}\right)$ for some $q<$ $p<c\left(\Sigma_{1,1}\right)$ with $q=2,3, \ldots, c\left(\Sigma_{1,2}\right)-1$. Then, $p \cdot g\left(\Sigma_{1,1}\right)$ $+\left(c\left(\Sigma_{1,1}\right)-p\right) \cdot g\left(\Sigma_{1,1}\right)=c\left(\Sigma_{1,1}\right) \cdot g\left(\Sigma_{1,1}\right)$, and we get $q \cdot g\left(\Sigma_{1,2}\right)+\left(c\left(\Sigma_{1,1}\right)-p\right) \cdot g\left(\Sigma_{1,1}\right)=x$. This gives $\Lambda_{2}(x) \neq \varnothing$, a contradiction. Therefore, we have $\left|\mathscr{B}^{*}(x)\right|=c\left(\Sigma_{1,1}\right)+c\left(\Sigma_{1,2}\right)-1$. As $\left|G_{I}(\Lambda)\right|=4, c\left(\Sigma_{1,1}\right)=3$ and $g\left(\Sigma_{1,2}\right)=2$ is the only possibility. This gives case (2).

Let $\left|\Lambda_{1}(x)\right|=1=\left|\Lambda_{2}(x)\right|$ and $\Lambda_{p}(x)=\varnothing, \forall p \geq 3$. Then, we can assume $\Sigma_{1} \in \Lambda_{1}(x)$ and $\Sigma_{2} \in \Lambda_{2}(x)$ are the only possible 1-representation and 2-representation of $x$, respectively. By (2) in Lemma 2 , we have $c\left(\Sigma_{2}\right)=(1,1)$. In this case, it is easy to see that $\mathscr{B}^{*}(x)=\left\{g\left(\Sigma_{1}\right), 2 g\left(\Sigma_{1}\right), \ldots\right.$, $\left.c\left(\Sigma_{1}\right) \cdot g\left(\Sigma_{1}\right), g_{1}\left(\Sigma_{2}\right), g_{2}\left(\Sigma_{2}\right)\right\}$. Then, $\left|\mathscr{B}^{*}(x)\right|=4$ gives $c\left(\Sigma_{1}\right)=2$ and we get case (3).
Lemma 5. Let $\Lambda=\langle\mathscr{A}\rangle$ be a numerical semigroup of embedding dimension $n \geq 2$. If $\left|G_{I}(\Lambda)\right|=5$, then $x$ is one of the following:

$$
\begin{aligned}
& \text { (1) } x=5 g\left(\Sigma_{1}\right) \text {. } \\
& \text { (2) } x=2 g_{1}\left(\Sigma_{2}\right)+g_{2}\left(\Sigma_{2}\right) \text {. } \\
& \text { (3) } x=g_{1}\left(\Sigma_{2,1}\right)+g_{2}\left(\Sigma_{2,1}\right) \text { and } x=g_{1}\left(\Sigma_{2,2}\right)+g_{2}\left(\Sigma_{2,2}\right) \text {. } \\
& \text { (4) } x=3 g\left(\Sigma_{1}\right) \text { and } x=g_{1}\left(\Sigma_{2}\right)+g_{2}\left(\Sigma_{2}\right) \text {. }
\end{aligned}
$$

Proof. Given that $\left|G_{I}(\Lambda)\right|=5$, then from Lemma 5, it follows that $x \in \Lambda$ satisfies one of the following conditions:

$$
\begin{aligned}
& \left|\Lambda_{1}(x)\right|=1 \text { and } \Lambda_{p}(x)=\varnothing, \forall p \geq 2 . \\
& \left|\Lambda_{2}(x)\right| \leq 2 \text { and } \Lambda_{p}(x)=\varnothing, \forall p \neq 2 . \\
& \left|\Lambda_{1}(x)\right|=1,\left|\Lambda_{2}(x)\right|=1 \text { and } \Lambda_{p}(x)=\varnothing, \forall p \geq 3 .
\end{aligned}
$$


These possibilities can be checked in a similar way as we did in Lemma 4 to get the required result.

\section{Graphs $G_{I}(\Lambda)$ with Metric Dimension 2}

Theorem 2. There are exactly 5 nonisomorphic graphs $G_{I}(\Lambda)$ with metric dimension 2.

We prove Theorem 2 in a sequence of following lemmas.

Lemma 6. There are exactly 2 nonisomorphic graphs $G_{I}(\Lambda)$ with 4 or less vertices and metric dimension 2.

Proof. It is trivial to note that no such graph exists for $\left|G_{I}(\Lambda)\right|=2,3$.

Now if $\left|G_{I}(\Lambda)\right|=4$, then from Lemma 4, we have the following possibilities:

(1) $x=4 g\left(\Sigma_{1}\right)$ with $\Lambda_{p}(x)=\varnothing, \forall p \geq 2$.

(2) $x=3 g\left(\Sigma_{1,1}\right)=2 g\left(\Sigma_{1,2}\right)$ with $\Lambda_{p}(x)=\varnothing, \forall p \geq 2$.

(3) $x=2 g\left(\Sigma_{1}\right)=g_{1}\left(\Sigma_{2}\right)+g_{2}\left(\Sigma_{2}\right)$ with $\Lambda_{p}(x)=\varnothing$, $\forall p \geq 3$.

If (1) holds, then $I=\Lambda \backslash \mathscr{B}^{*}\left(4 g\left(\Sigma_{1}\right)\right)$, and therefore, $G_{I}(\Lambda)$ is isomorphic to the graph given in Figure 1. So metric dimension of $G_{I}(\Lambda)$ is 2 .

Now if (2) or (3) holds, then either $I=\Lambda \backslash \mathscr{B}^{*}\left(3 g\left(\Sigma_{1,1}\right)\right)$ or $I=\Lambda \backslash \mathscr{B}^{*}\left(2 g\left(\Sigma_{1}\right)\right)$. In both cases, $G_{I}(\Lambda)$ is isomorphic to the graph given in Figure 2, and therefore, metric dimension of $G_{I}(\Lambda)$ is 2 .

Lemma 7. There are exactly 3 nonisomorphic graphs $G_{I}(\Lambda)$ with 5 vertices and metric dimension 2.

Proof. If $\left|G_{I}(\Lambda)\right|=5$, then from Lemma 5, we have the following possibilities:

(1) $x=5 g\left(\Sigma_{1}\right)$ with $\Lambda_{p}(x)=\varnothing, \forall p \geq 2$.

(2) $x=2 g_{1}\left(\Sigma_{2}\right)+g_{2}\left(\Sigma_{2}\right)$ with $\Lambda_{p}(x)=\varnothing, \forall p \neq 2$.

(3) $x=g_{1}\left(\Sigma_{2,1}\right)+g_{2}\left(\Sigma_{2,1}\right)=g_{1}\left(\Sigma_{2,2}\right)+g_{2}\left(\Sigma_{2,2}\right) \quad$ with $\Lambda_{p}(x)=\varnothing, \forall p \neq 2$.

(4) $x=3 g\left(\Sigma_{1}\right) \quad$ and $\quad x=g_{1}\left(\Sigma_{2}\right)+g_{2}\left(\Sigma_{2}\right) \quad$ with $\Lambda_{p}(x)=\varnothing, \forall p \geq 3$.

If (1) holds, then $I=\Lambda \backslash \mathscr{B}^{*}\left(5 g\left(\Sigma_{1}\right)\right)$, and therefore, $G_{I}(\Lambda)$ is isomorphic to the graph given in Figure 3 .

Now, if (2) holds, then $I=\Lambda \backslash \mathscr{B}^{*}\left(2 g_{1}\left(\Sigma_{2}\right)+g_{2}\left(\Sigma_{2}\right)\right)$, and therefore, $G_{I}(\Lambda)$ is isomorphic to the graph given in Figure 4.

If (3) or (4) holds, then $I=\Lambda \backslash \mathscr{B}^{*}\left(g_{1}\left(\Sigma_{2,1}\right)+g_{2}\left(\Sigma_{2,1}\right)\right)$ or $I=\Lambda \backslash \mathscr{B}^{*}\left(3 g\left(\Sigma_{1}\right)\right)$. In both cases, $G_{I}(\Lambda)$ is isomorphic to the graph given in Figure 5.

For all these 3 cases, one can easily show that metric dimension of $G_{I}(\Lambda)$ is 2 .

Finally, it is required to check all the graphs $G_{I}(\Lambda)$ of order six having metric dimension 2 . Binyamin et al. [34] proved that if $\left|G_{I}(\Lambda)\right|=6$, then $G_{I}(\Lambda)$ is isomorphic to one of the graphs given in Table 1 . Now, it is easy to see that all the graphs given in Table 1 have metric dimension 3.

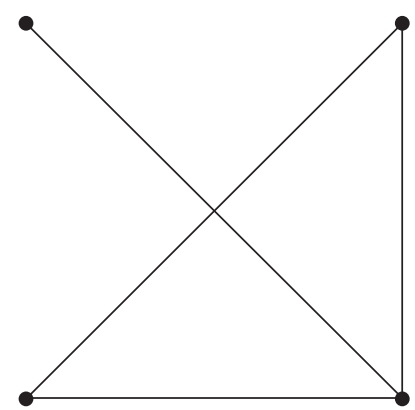

FIgURE 1: Graphs $G_{I}(\Lambda)$ When $x=4 g\left(\Sigma_{1}\right)$ with $\Lambda_{p}(x)=0$, $\forall p \geq 2$.

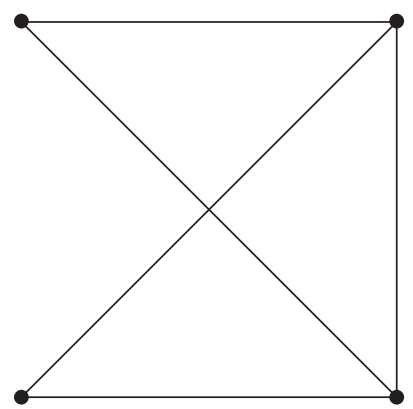

Figure 2: Graph $G_{I}(\Lambda)$ for the remaining two cases.

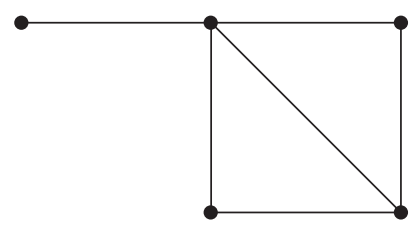

Figure 3: Graphs $G_{I}(\Lambda)$ When $x=5 g\left(\Sigma_{1}\right)$ with $\Lambda_{p}(x)=0$, $\forall p \geq 2$.

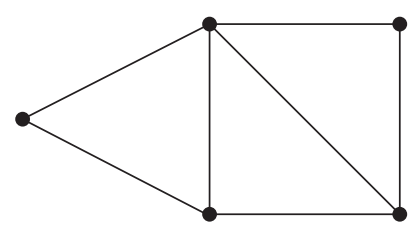

FIgure 4: Graphs $G_{I}(\Lambda)$ When $x=2 g\left(\Sigma_{2}\right)$ with $\Lambda_{p}(x)=0$, $\forall p \neq q 2$.

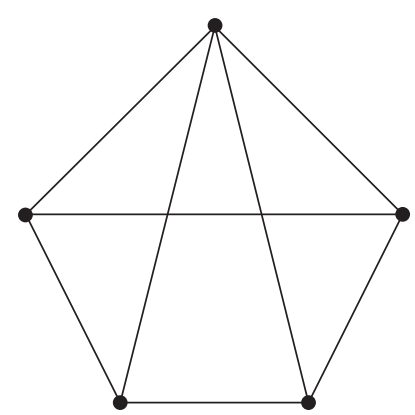

Figure 5: Graph $G_{I}(\Lambda)$ for the remaining two cases. 
TABLe 1: Graph $G_{I}(\Lambda)$ of order 6 up to isomorphism.

1

$(1,2,3,3,4,5)$

2

$(2,3,3,4,5,5)$

3

$(2,3,4,4,4,5)$

4

$(3,3,4,4,5,5)$

5

$(3,4,4,4,4,5)$

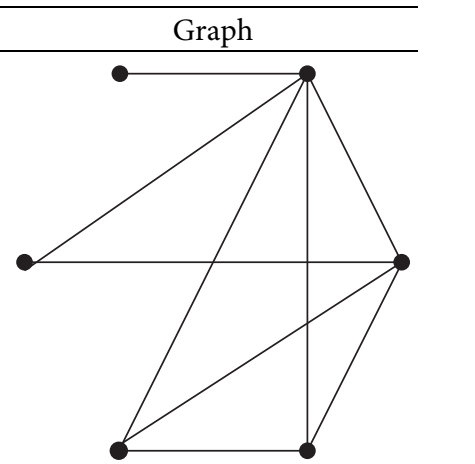

6

$(4,4,4,4,5,5)$

TABle 1: Continued.

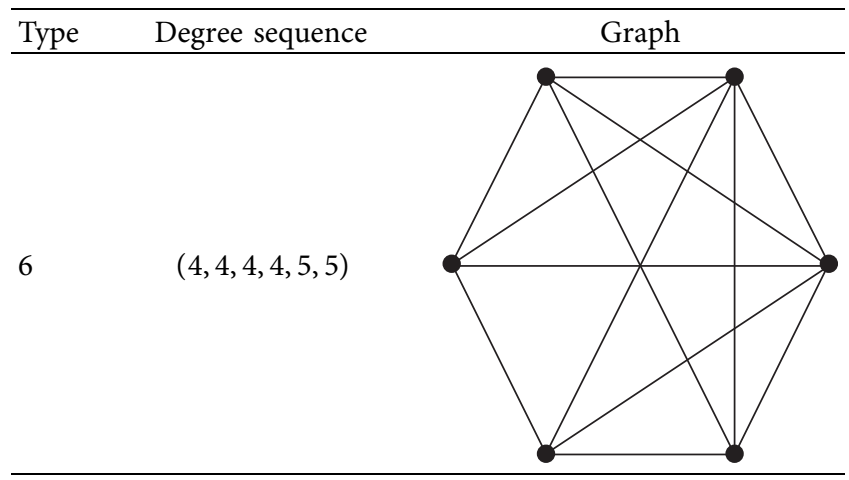

\section{Data Availability}

No data are required for the study.

\section{Conflicts of Interest}

The authors declare that they have no conflicts of interest.

\section{Acknowledgments}

This work was supported by the National Key R and D Program of China (no. 2018YFB1005100), Innovation Projects in Universities of Guangdong Province (no. 2020KTSCX21), and Development Project of Young Scientific and Technological Talents in Higher Education of Guizhou Province (no. QianJiaoHe KY[2021]250).

\section{References}

[1] M. Afkhami and K. Khashyarmanesh, "The intersection graph of ideals of a lattice," Note di Matematica, vol. 34, no. 2, pp. 135-143, 2014.

[2] D. F. Anderson and P. S. Livingston, "The zero-divisor graph of a commutative ring," Journal of Algebra, vol. 217, no. 2, pp. 434-447, 1999.

[3] D. F. Anderson and A. Badawi, "The total graph of a commutative ring," Journal of Algebra, vol. 320, no. 7, pp. 2706-2719, 2008.

[4] A. Badawi, "On the annihilator graph of a commutative ring," Communications in Algebra, vol. 42, no. 1, pp. 108-121, 2014.

[5] M. Behboodi and Z. Rakeei, "The annihilating-ideal graph of commutative rings I," Journal of Algebra and Its Applications, vol. 10, no. 4, pp. 727-739, 2011.

[6] H. R. Maimani, M. Salimi, A. Sattari, and S. Yassemi, "Comaximal graph of commutative rings," Journal of Algebra, vol. 319, no. 4, pp. 1801-1808, 2008.

[7] D. F. Anderson and S. B. Mulay, "On the diameter and girth of a zero-divisor graph," Journal of Pure and Applied Algebra, vol. 210, no. 2, pp. 543-550, 2007.

[8] T. Tamizh Chelvam and K. Selvakumar, "Central sets in annihilating-ideal graph of a commutative ring," Journal of 
Combinatorial Mathematics and Combinatorial Computer, vol. 88, pp. 277-288, 2014.

[9] T. Tamizh Chelvam and K. Selvakumar, "Domination in the directed zero-divisor graph of ring of matrices," Journal of Combinatorial Mathematics and Combinatorial Computer, vol. 91, pp. 155-163, 2014.

[10] S. Akbari, H. R. Maimani, and S. Yassemi, "When a zero-divisor graph is planar or a complete r-partite graph," Journal of Algebra, vol. 270, no. 1, pp. 169-180, 2003.

[11] I. Beck, "Coloring of commutative rings," Journal of Algebra, vol. 116, pp. 208-226, 1998.

[12] R. F. Bailey and P. J. Cameron, "Base size, metric dimension and other invariants of groups and graphs," Bulletin of the London Mathematical Society, vol. 43, no. 2, pp. 209-242, 2011.

[13] S. Pirzada and R. Raja, "On the metric dimension of a zero-divisor graph," Communications in Algebra, vol. 45, no. 4, pp. 1399-1408, 2017.

[14] P. J. Slater, "Leaves of trees," Congressus Numerantium, vol. 14, pp. 549-559, 1975.

[15] F. Harary and R. A. Melter, "On the metric dimension of a graph,” Ars Combinatoria, vol. 2, pp. 191-195, 1976.

[16] S. Khuller, B. Raghavachari, and A. Rosenfeld, "Localization in graphs," Technical report CS-TR- 3326, University of Maryland at College Park, College Park, MD, USA, 1994.

[17] P. J. Cameron and J. H. Van Lint, "Designs, graphs, codes and their links," in London Mathematical Society Student TextsCambridge University Press, Cambridge, UK, 1991.

[18] G. Chartrand, L. Eroh, M. A. Johnson, and O. R. Oellermann, "Resolvability in graphs and the metric dimension of a graph," Discrete Applied Mathematics, vol. 105, no. 1-3, pp. 99-113, 2000.

[19] A. Sebo and E. Tannier, "On metric generators of graphs," Mathematics of Operations Research, vol. 29, pp. 383-393, 2004.

[20] J. C. Rosales and P. A. Garcia-Sanchez, "Numerical semigroups," Note di Matematica, vol. 34, pp. 135-143, 2014.

[21] V. Soleymanivarniab, A. Tehranian, and R. Nikandish, "The metric dimension of annihilator graphs of commutative rings," Journal of Algebra and Its Applications, vol. 19, no. 5, 2020.

[22] R. F. Bailey, J. Cáceres, D. Garijo et al., "Resolving sets for Johnson and kneser graphs," European Journal of Combinatorics, vol. 34, no. 4, pp. 736-751, 2013.

[23] F. Ali, M. Salman, and S. Huang, "On the commuting graph of dihedral group," Communications in Algebra, vol. 44, no. 6, pp. 2389-2401, 2016.

[24] D. Dolzan, "The metric dimension of the total graph of a finite commutative ring," Canadian Mathematical Bulletin, vol. 59, no. 4, pp. 748-759, 2016.

[25] A. N. A. Koam and A. Ahmad, "Barycentric subdivision of cayley graphs with constant edge metric dimension," IEEE Access, vol. 8, no. 1, pp. 80624-80628, 2020.

[26] M. B. Ali Ahmad, "Saba sultan, on metric dimension and minimal doubly resolving sets of harary graph," $A M U C$, vol. 89, no. 1, pp. 123-129, 2020.

[27] A. N. A. Koam, A. Ahmad, and A. Haider, "Radio number associated with zero divisor graph," Mathematics, vol. 8, no. 12 , p. $2187,2020$.

[28] Z. Shao, S. M. Sheikholeslami, P. Wu, and J.-B. Liu, "The metric dimension of some generalized Petersen graphs," Discrete Dynamics in Nature and Society, vol. 2018, Article ID 4531958, 10 pages, 2018.
[29] Z. Shao, P. Wu, E. Zhu, and L. Chen, "On metric dimension in some hex derived networks," Sensors, vol. 19, no. 1, p. 94, 2019.

[30] E. Zhu, A. Taranenko, Z. Shao, and J. Xu, "On graphs with the maximum edge metric dimension," Discrete Applied Mathematics, vol. 257, pp. 317-324, 2019.

[31] M. A. Binyamin, H. M. A. Siddiqui, N. M. Khan, A. Aslam, and Y. Rao, "Characterization of graphs associated with numerical semigroups," Mathematics, vol. 7, no. 6, p. 557, 2019.

[32] P. Xu, M. A. Binyamin, A. Aslam, W. Ali, H. Mahmood, and H. Zhou, "Characterization of graphs associated to the ideal of the numerical semigroups," Journal of Mathematics, vol. 14, 2020.

[33] V. Barucci, "Decompositions of ideals into irreducible ideals in numerical semigroups," Journal of Commutative Algebra, vol. 2, no. 3, pp. 281-294, 2010.

[34] M. Ahsan Binyamin, W. Ali, A. Aslam, and H. Mahmood, "Classification of planar graphs associated to the ideal of the numerical semigroup," 2012, https://arxiv.org/abs/2012. 10434. 